\title{
The origin of optical emission from super-Eddington accreting Active Galactic Nuclei: The case of Ton S 180
}

\author{
T. Kawaguchi ${ }^{1,2}$, A. Pierens ${ }^{1}$, and J.-M. Huré ${ }^{1,3}$ \\ ${ }^{1}$ LUTh/Observatoire de Paris-Meudon et CNRS UMR 8102, Place Jules Janssen, 92195 Meudon Cedex, France \\ e-mail: Toshihiro.Kawaguchi@obspm.fr; Arnaud.Pierens@obspm.fr; Jean-Marc.Hure@obspm.fr \\ 2 Postdoctoral Fellow of the Japan Society for the Promotion of Science \\ 3 Université Paris 7 Denis Diderot, 2 place Jussieu, 75251 Paris Cedex 05, France
}

Received 2 May 2003 / Accepted 17 October 2003

\begin{abstract}
Self-gravitating accretion discs have only been studied in a few nearby objects using maser spots at the parsec-scale. We find a new spectral window for observing the self-gravitating accretion disc in super-Eddington accreting Active Galactic Nuclei (AGNs). This window is determined by calculating the outermost radius $\left(r_{\mathrm{sg}}\right)$ of a non self-gravitating disc and the corresponding emission wavelength $\left(\lambda_{\mathrm{sg}}\right)$ as a function of various disc parameters. We find that $\lambda_{\text {sg }}$ reaches $\sim 4000 \AA$ for $\alpha=0.1$, when $\dot{M} \gtrsim 70\left(M_{\mathrm{BH}} / 10^{7} M_{\odot}\right)^{-1} L_{\mathrm{Edd}} / c^{2}$ (where $\alpha, \dot{M}, M_{\mathrm{BH}}$ and $L_{\mathrm{Edd}}$ are, respectively, the viscosity parameter, gas accretion rate onto the central black hole $(\mathrm{BH})$, the $\mathrm{BH}$ mass and the Eddington luminosity). Moreover, $\lambda_{\mathrm{sg}}$ is as small as $\sim 1500 \AA$ for $\alpha=0.001$, which is the smallest $\alpha$ case in this study. Therefore, the window for observing the self-gravitating part of an AGN accretion disc is from $\sim 2 \mu \mathrm{m}$ to $\lambda_{\text {sg }}$. Incidentally, $r_{\mathrm{sg}}$ can be less than the photon trapping radius for $\dot{M} \gtrsim 10^{3.3} L_{\mathrm{Edd}} / c^{2}$. Namely, a self-gravitating, optically-thick, advection-dominated accretion disc is expected to appear in the extremely high accretion rate regime.

Next, we demonstrate that the Mid-Infrared to X-ray spectrum of a bright, well-studied Narrow-Line Seyfert 1 galaxy, Ton S 180, is indeed well fitted by the spectrum arising from the following three components: an inner slim disc (with a corona), an outer, self-gravitating non-Keplerian disc and a dusty torus. The total mass, $\mathrm{BH}$ mass plus the entire disc mass, is found to be about (1.4-8.0) $M_{\mathrm{BH}}$. If the surface density varies with radius $r$ in proportion to $r^{-0.6}$, the total mass is consistent with the central mass estimated by $\mathrm{H} \beta$ and [O III] widths.
\end{abstract}

Key words. accretion, accretion disks - radiation mechanisms: thermal - galaxies: active - galaxies: individual: Ton S 180 galaxies: nuclei - galaxies: Seyfert

\section{Introduction}

The Optical/UV/X-ray emission from Active Galactic Nuclei (AGNs) is thought to arise from a hot accretion disc around a super-massive Black Hole (BH; e.g., Mushotzky et al. 1993; Koratkar \& Blaes 1999), while the Mid- and Near Infrared (IR) radiation comes from a cold, dusty torus (e.g., Telesco et al. 1984; Thatte et al. 1997; Pier \& Krolik 1993). Disc self-gravity can generally be ignored in the innermost region where the high energy spectrum is formed. At large distances (typically $\geq 10^{3}-10^{4}$ Schwarzschild radii, depending on accretion parameters), the disc mass is expected to play a significant role. Disc self-gravity has been tested only at the parsec-scale via maser spots detected in a few nearby objects (e.g. Nakai et al. 1993; Miyoshi et al. 1995; Huré 2002; Lodato \& Bertin 2003).

Narrow Line Seyfert 1 galaxies (NLS1s) and their high luminosity analogue, Narrow-Line QSOs, are supposed to have high accretion rates among the AGN population (e.g., Brandt \& Boller 1998; Mineshige et al. 2000). The gas accretion

Send offprint requests to: T. Kawaguchi, e-mail: Toshihiro.Kawaguchi@obspm.fr rate $\dot{M}$ for those objects is expected to be comparable to or larger than $10 L_{\mathrm{Edd}} / c^{2}$, where $L_{\mathrm{Edd}}$ is the Eddington luminosity. For such super-Eddington accretion rates, the outer edge of the non self-gravitating part of the accretion disc radiates optical continuum emission ( $\sim 4000 \AA)$ for a certain parameter set (Kawaguchi 2003). Thus, they are potentially good candidates to study the self-gravitating part of the disc observationally at longer wavelengths (Collin et al. 2002; Kawaguchi 2003).

In this paper, we qualitatively describe the wavelength corresponding to the emission from the outer edge of a non selfgravitating disc for various accretion parameter sets. This is done in Sect. 2. We present in Sect. 3 a Mid-IR to X-ray spectral modeling of a bright, well-studied NLS1, Ton S 180 (PHL912), for which numerous, multi-waveband observations are available (e.g., Wisotzki et al. 1995; Turner et al. 2002; Vaughan et al. 2002). In Sect. 4, several discussions are presented. The final section is devoted to a summary.

\section{Critical radius and critical wavelength}

In this section, we calculate the radius of the outer edge of the non self-gravitating $\operatorname{slim} \operatorname{disc}\left(r_{\mathrm{sg}}\right)$, i.e. inner edge of the 


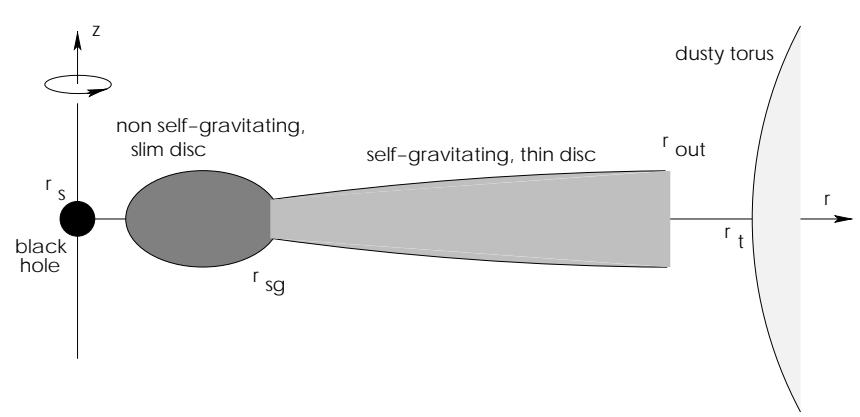

Fig. 1. Schematic configuration (not to scale) of the three relevant components surrounding the black hole: a slim disc, a self-gravitating disc and a torus. For Ton S 180 (Sect. 3), the characteristic radii are found to be the followings: $r_{\mathrm{sg}} \simeq 3 \times 10^{3} r_{\mathrm{Sch}}, r_{\text {out }} \simeq 3 \times 10^{4} r_{\mathrm{Sch}}$ and $r_{\mathrm{t}} \simeq 3 \times 10^{5} r_{\text {Sch }}$. The broad-line region is located at $r \simeq 1.4 \times 10^{5} r_{\text {Sch }}$ (Sect. 4).

self-gravitating disc, as a function of the $\mathrm{BH}$ mass $M_{\mathrm{BH}}, \dot{M}$ and viscosity parameter $\alpha$. We will then show the corresponding wavelength $\left(\lambda_{\mathrm{sg}}\right)$ that is relevant to emission from $r_{\mathrm{sg}}$. We define $M_{7}$ for $M_{\mathrm{BH}}$ in the unit of $10^{7} M_{\odot}$.

Figure 1 illustrates the configuration considered in this study.

\subsection{Critical radius}

For super-Eddington accretion rates (i.e. $\dot{M} \gtrsim 10 L_{\mathrm{Edd}} / \mathrm{c}^{2}$ ), an optically-thick, advection-dominated regime appears (Abramowicz et al. 1988). Inside a certain radius $r_{\text {trap }} \sim 0.5 \dot{M}\left(L_{\text {Edd }} / c^{2}\right)^{-1} r_{\text {Sch }}$ (Begelman \& Meier 1982; Kawaguchi 2003), the timescale for photon diffusion is larger than the accretion timescale, and thus most of the photons emitted inside the flow are trapped within it. Thus, advection cooling becomes dominant over radiative cooling at $r<r_{\text {trap }}$.

We define $r_{\mathrm{sg}}$ as the radius where the mass density in the disc mid-plane ( $\rho_{\text {mid-plane }}$ ) equals $\rho_{\text {sg }}$. Here, $\rho_{\text {sg }}$ is a critical density above which self-gravity of the disc must be considered:

$\rho_{\mathrm{sg}}=\Omega_{\mathrm{K}}^{2} /(4 \pi \mathrm{G})=2.2 M_{7}^{-2}\left(r / 3 r_{\mathrm{Sch}}\right)^{-3} \mathrm{~g} / \mathrm{cm}^{3}$,

where $\Omega_{\mathrm{K}}$ is the Keplerian rotation frequency (e.g., Goldreich \& Lynden-Bell 1965; Huré 1998). Inside $r_{\mathrm{sg}}, \rho_{\text {mid-plane }}$ is smaller than $\rho_{\mathrm{sg}}$, thus the disc self-gravity is negligible there.

Many authors have tried to derive $r_{\text {sg }}$ (e.g., Shore \& White 1982). Actually, $r_{\mathrm{sg}}\left(M_{\mathrm{BH}}, \dot{M}, \alpha\right)$ derived and used in this study is qualitatively consistent with that in Huré (1998).

In order to derive $r_{\mathrm{sg}}$, we use analytical formulae for the mass density $\left(\rho_{\text {mid-plane }}\right)$ given for a Newtonian gravitational potential (e.g., Kato et al. 1998). We confirm that these standard-disc formulae for various physical quantities are consistent with numerical computations (Kawaguchi 2003) within a factor of $\sim 1.5$ at any radius larger than $r_{\text {trap }}$. In other words, the flow behaves like a standard disc (Shakura \& Sunyaev 1973) outside $r_{\text {trap }}$, even for a super-Eddington accretion rate.

In a standard disc, the following three regions must be distinguished according to the sources of the pressure and opacity (e.g., Shakura \& Sunyaev 1973; Kato et al. 1998). In which region $r_{\text {sg }}$ appears is determined by $\dot{M}$ (see Fig. 2).

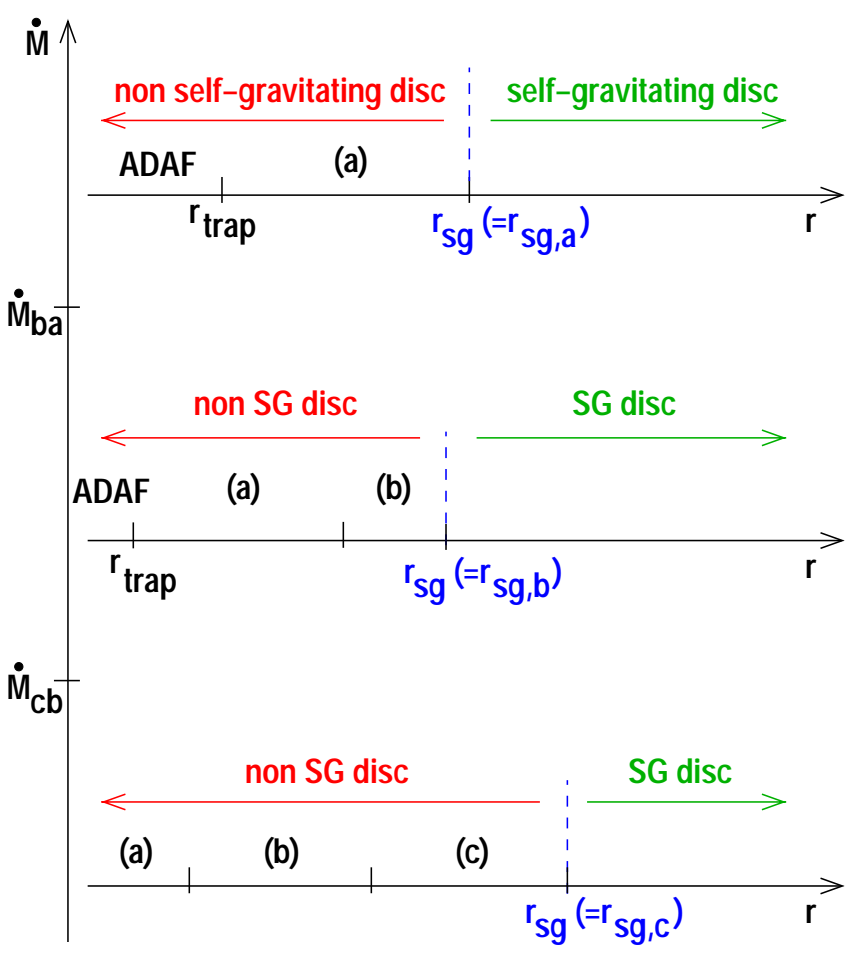

Fig. 2. Classification of the region where $r_{\mathrm{sg}}$ appears (Sect. 2.1). Region (c) harbors $r_{\mathrm{sg}}$ when $\dot{M}$ is less than $\dot{M}_{\mathrm{cb}}$, while regions (b) and (a) are, respectively, in charge of $r_{\mathrm{sg}}$ when $\dot{M}$ is smaller and larger than $\dot{M}_{\text {ba }}$. Around the radius $r_{\text {trap }}$, the flow undergoes a change from radiation cooling-dominated regime to advection-dominated regime (optically-thick ADAF).

- an outer region (c), where the gas pressure and absorption opacity dominate over radiation pressure and electronscattering opacity, respectively. With a relatively low accretion rate, i.e. $\dot{M} \lesssim \dot{M}_{\mathrm{cb}}=2 M_{7}^{-1}(\alpha / 0.1)^{7 / 13} L_{\mathrm{Edd}} / c^{2}, r_{\mathrm{sg}}$ appears at the outer region (c):

$r_{\mathrm{sg}, \mathrm{c}}=6700\left(\frac{\alpha}{0.1}\right)^{28 / 45} M_{7}^{-52 / 45}\left(\frac{\dot{M}}{L_{\mathrm{Edd}} / c^{2}}\right)^{-22 / 45} r_{\mathrm{Sch}}$,

- a middle region (b) where gas pressure and electronscattering opacity are dominant. For a moderately high accretion rate, i.e. $\dot{M} \lesssim \dot{M}_{\mathrm{ba}}=70 M_{7}^{-1}(\alpha / 0.1)^{0.4} L_{\mathrm{Edd}} / c^{2}, r_{\mathrm{sg}}$ is given by:

$r_{\mathrm{sg}, \mathrm{b}}=5700\left(\frac{\alpha}{0.1}\right)^{14 / 27} M_{7}^{-26 / 27}\left(\frac{\dot{M}}{L_{\mathrm{Edd}} / c^{2}}\right)^{-8 / 27} r_{\mathrm{Sch}}$,

- an inner region (a) which is described by radiation pressure and electron-scattering opacity. For a higher accretion rate $\left(\dot{M} \gtrsim \dot{M}_{\text {ba }}\right), r_{\text {sg }}$ is described as

$$
r_{\mathrm{sg}, \mathrm{a}}=250\left(\frac{\alpha}{0.1}\right)^{2 / 9} M_{7}^{-2 / 9}\left(\frac{\dot{M}}{L_{\mathrm{Edd}} / c^{2}}\right)^{4 / 9} r_{\mathrm{Sch}} .
$$

Given $M_{\mathrm{BH}}$ and $\dot{M}$, the critical radius $r_{\mathrm{sg}}$ can be written as $\operatorname{Max}\left(r_{\mathrm{sg}, \mathrm{c}}, r_{\mathrm{sg}, \mathrm{b}}, r_{\mathrm{sg}, \mathrm{a}}\right)$, for the whole range of accretion rates. Instead of a piece-wise function, we employ a simple formula: $r_{\mathrm{sg}}=\left(r_{\mathrm{sg}, \mathrm{a}}{ }^{3}+r_{\mathrm{sg}, \mathrm{b}}{ }^{3}+r_{\mathrm{sg}, \mathrm{c}}{ }^{3}\right)^{1 / 3}$. Figure 3 (upper panels) shows $r_{\mathrm{sg}}$ for various $M_{\mathrm{BH}}, \dot{M}$ and $\alpha$. Until the accretion rate reaches $\dot{M}_{\mathrm{ba}}$, 


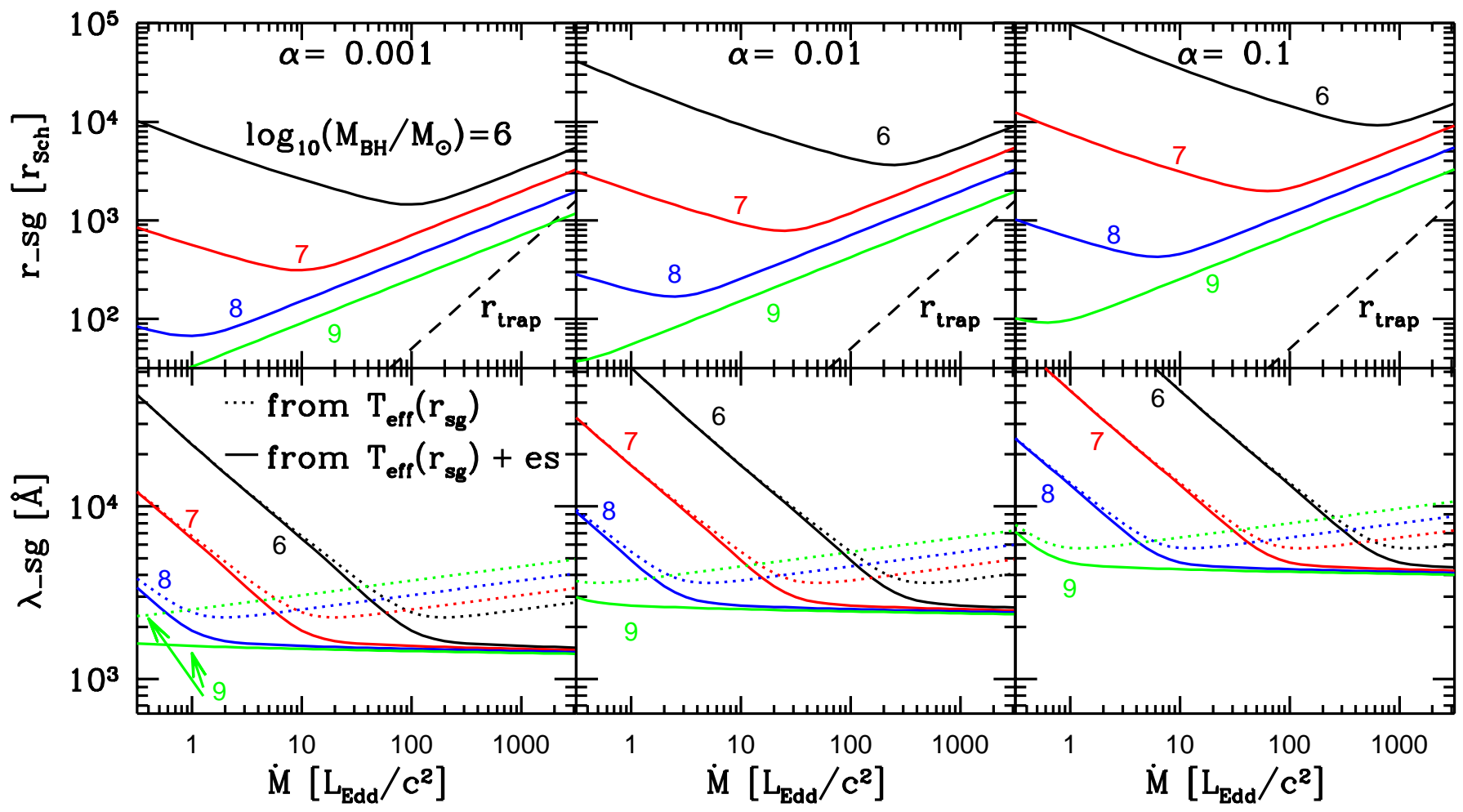

Fig. 3. Critical radius and wavelength $\left[r_{\mathrm{sg}}\right.$ (upper) and $\lambda_{\mathrm{sg}}$ (lower)] for various $M_{\mathrm{BH}}, \dot{M}$ and $\alpha$. Here, $r_{\text {trap }}$ (dashed lines in the upper panels) is the radius inside which photon trapping plays a role. The dotted lines in the lower panel are computed based on the assumption that the radiation from $r_{\mathrm{sg}}$ is blackbody, while solid lines take the effect of electron scattering (modified blackbody) into account. If $\lambda_{\mathrm{sg}} \lesssim 2 \mu \mathrm{m}$, it means that we are able to observe the self-gravitating accretion disc as Near-IR/optical continuum radiation.

$r_{\mathrm{sg}}$ decreases with an increasing $\dot{M}$, while $r_{\mathrm{sg}}$ changes the sign of its $\dot{M}$-dependency at $\dot{M} \sim \dot{M}_{\text {ba }}$.

We find that with $\dot{M} \gtrsim 10^{3.3}(\alpha / 0.001)^{0.4}$ $\left(M_{\mathrm{BH}} / 10^{9} M_{\odot}\right)^{-0.4} L_{\mathrm{Edd}} / c^{2}, r_{\mathrm{sg}}$ can be less than $r_{\text {trap }}$. The above expressions are no longer valid in the regime in which $r_{\mathrm{sg}}<r_{\text {trap }}$. In that case, a self-gravitating, optically-thick, advection-dominated accretion disc appears. Therefore, we restrict ourselves to accretion rates lower than $10^{3.5} L_{\mathrm{Edd}} / c^{2}$, in order to ensure that $r_{\text {sg }} \gtrsim r_{\text {trap }}$.

\subsection{Critical wavelength}

Next, we evaluate the effective temperature at $r_{\mathrm{sg}}$, and subsequently a critical wavelength $\lambda_{\mathrm{sg}}$ corresponding to the emission from $r_{\text {sg }}$. At $r>r_{\text {trap }}$, the effective temperature $T_{\text {eff }}$ at radius $r$ is given by

$T_{\text {eff }}(r)=6.2 \times 10^{5} M_{7}^{-1 / 4}\left(\frac{\dot{M}}{L_{\mathrm{Edd}} / c^{2}}\right)^{1 / 4}\left(\frac{r}{r_{\mathrm{Sch}}}\right)^{-3 / 4} \mathrm{~K}$.

The simplest procedure to estimate $\lambda_{\mathrm{sg}}$ is to use the Wien displacement law assuming that the emission is close to a blackbody radiation: namely $\lambda_{\mathrm{sg}} \simeq h c / 4 k T_{\text {eff }}$ (see Rybicki \& Lightman 1979). It follows that the critical wavelength is

$\lambda_{\mathrm{sg}}=\frac{h c}{4 k T_{\mathrm{eff}}\left(r_{\mathrm{sg}}\right)}=0.36\left[\frac{T_{\mathrm{eff}}\left(r_{\mathrm{sg}}\right)}{\mathrm{K}}\right]^{-1} \mathrm{~cm}$.

The results are shown by the dotted lines in Fig. 3 (lower panels).
The effects of electron scattering (opacity and Comptonization) must be considered in studying the emergent spectra from a super-Eddington accreting disc (Shimura \& Manmoto 2003; Wang \& Netzer 2003; Kawaguchi 2003). Comptonization is important at radius less than $\sim 300 r_{\text {Sch }}$ for $\dot{M} \lesssim 1000 L_{\mathrm{Edd}} / c^{2}$, and thus it has no effect on the emission beyond $r_{\mathrm{sg}}$. On the other hand, the ratio of electronscattering opacity to absorption opacity is larger than unity at $r \lesssim 2500\left[\dot{M} /\left(L_{\text {Edd }} / c^{2}\right)\right]^{2 / 3} r_{\text {Sch }}$ (see Kato et al. 1998), and thus the effect of electron-scattering opacity is still important at $r_{\mathrm{sg}}$.

Due to the electron-scattering opacity, the emergent spectrum $\left(I_{v}\right)$ is distorted towards higher energy (i.e. shorter wavelength), and is close to the so called modified blackbody (Rybicki \& Lightman 1979; Czerny \& Elvis 1987; Wandel \& Petrosian 1988):

$$
I_{v}=\frac{2 B_{v}\left(T_{\mathrm{eff}}\right)}{1+\sqrt{\left(\kappa_{\mathrm{abs}, v}+\kappa_{\mathrm{es}}\right) / \kappa_{\mathrm{abs}, v}}} .
$$

Here, $B_{v}, \kappa_{\mathrm{abs}, v}$ and $\kappa_{\mathrm{es}}\left(=0.4 \mathrm{~cm}^{2} / \mathrm{g}\right)$ are, respectively, the Planck function, absorption opacity and electron-scattering opacity. The spectral shift due to this effect is roughly estimated as follows (Madej 1974):

$$
\Delta \log _{10} \lambda_{\mathrm{sg}} \simeq \frac{1}{8} \log _{10}\left[\frac{\kappa_{\mathrm{abs}}\left(r_{\mathrm{sg}}\right)}{\kappa_{\mathrm{abs}}\left(r_{\mathrm{sg}}\right)+\kappa_{\mathrm{es}}\left(r_{\mathrm{sg}}\right)}\right],
$$

where $\kappa_{\mathrm{abs}}$ is the Rosseland mean opacity for absorption (bound-bound, bound-free and free-free processes). In assessing $\kappa_{\mathrm{abs}}$, we take 30 times the free-free absorption 


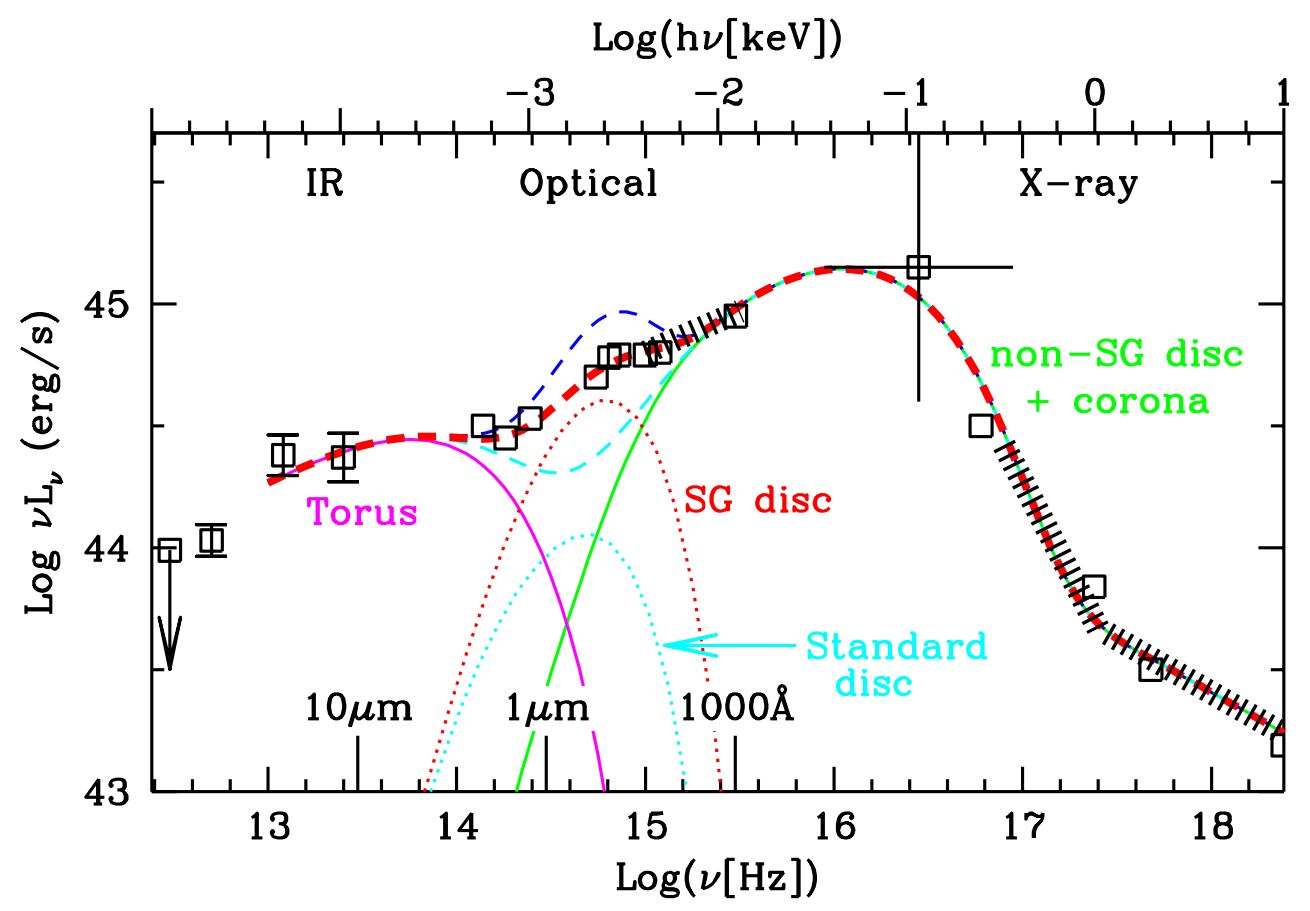

Fig. 4. Mid-IR to X-ray spectral modeling of Ton S 180. Open squares and thick striped lines are the observed data and the power-law fits described in Turner et al. (2002). Two solid lines are the spectral components of the dusty torus (left) and the non self-gravitating disc with a corona (right; $M_{\mathrm{BH}}=10^{6.8} M_{\odot}, \dot{M}=1000 L_{\mathrm{Edd}} / c^{2}$, and $\alpha=0.002$ ). Two dotted lines mean the spectrum (from $r_{\mathrm{sg}}<r<r_{\mathrm{out}}$ ) of the self-gravitating disc for $\gamma=-0.6$ (where $\Sigma \propto r^{\gamma}$ ) and $\alpha_{\text {out }}=0.02$ (upper; one of the successful fits shown in Table 2) and that of a Keplerian, standard disc (lower). Total spectra of those three components are indicated by dashed lines: thick line is the spectrum with the self-gravitating disc mentioned above, while the case with a standard disc is indicated by a lower curve. The upper dashed line exhibits a total spectrum with a different parameter set for the outer self-gravitating disc $\left(\gamma=0, \alpha_{\text {out }}=0.02\right)$.

opacity, as is done in Czerny \& Elvis (1987) and Kawaguchi (2003):

$\kappa_{\mathrm{abs}}=30 \kappa_{\mathrm{ff}}$

with the free-free absorption opacity, $\kappa_{\mathrm{ff}}$, as follows

$\kappa_{\mathrm{ff}}=6.4 \times 10^{22}\left(\frac{\rho_{\text {mid-plane }}}{\mathrm{g} \mathrm{cm}^{-3}}\right)\left(\frac{T_{\text {mid-plane }}}{\mathrm{K}}\right)^{-3.5} \mathrm{~cm}^{2} / \mathrm{g}$.

Here, $T_{\text {mid-plane }}$ is the temperature at the mid-plane of the disc (Kato et al. 1998).

Finally, $\lambda_{\text {sg }}$ modified from Eq. (6) by Eq. (8) are plotted in the lower panels of Fig. 3 as solid lines. As far as $\dot{M}<\dot{M}_{\text {ba }}, \lambda_{\mathrm{sg}}$ is roughly proportional to $\dot{M}^{-0.5}$. For $\dot{M}>\dot{M}_{\text {ba }}$ and $\alpha=0.1, \lambda_{\mathrm{sg}}$ reaches and stays at $\sim 4000 \AA$, and interestingly it can be as small as $\sim 1500 \AA$ for the lowest $\alpha$ case here (i.e. $\alpha=0.001$ ). Because of dust sublimation at $T \gtrsim 1500 \mathrm{~K}$, emission of dust falls within the Mid-IR-Near-IR spectral range and can not contribute to the optical component (see Sect. 3.3). Therefore, emission from $\sim 2 \mu \mathrm{m}$ to $\lambda_{\mathrm{sg}}$ arises from the self-gravitating part of the super-Eddington accreting disc. In other words, this is a discovery of a spectral window for observing the selfgravitating disc, which has solely been studied by maser spots.

We note that $\lambda_{\mathrm{sg}}$ for $M_{\mathrm{BH}} \gtrsim 10^{9} M_{\odot}$ is also small even with sub-Eddington accretion rates. In the sub-Eddington regime, however, heating by central radiation onto an outer region at $r \sim r_{\mathrm{sg}}$ may not be negligible. An uncertainty of physical quantities (such as the gas density) in the outer region of the disc (e.g., Hōshi \& Inoue 1988) will lead to uncertainties of $r_{\mathrm{sg}}$ and $\lambda_{\mathrm{sg}}$. On the contrary, a super-Eddington accreting disc is not flared, i.e. it has a maximum of the aspect ratio at $r \sim(5-100) r_{\text {Sch }}$ (Kawaguchi 2003), when such irradiation is considered to be less important. For this reason, $r_{\mathrm{sg}}$ and $\lambda_{\mathrm{sg}} \mathrm{ob}-$ tained for high $\dot{M}$ are more reliable than for the sub-Eddington cases. Therefore, we concentrate on the super-Eddington cases.

\section{Spectral calculations}

We now demonstrate that the broad-band spectrum of a bright, well-studied NLS1, Ton S 180, is indeed well fitted by the spectrum arising from three components: a slim disc (with a coronal, hard X-ray spectrum), a self-gravitating non-Keplerian disc and a dusty torus. The configuration we consider is schematically shown in Fig. 1.

The Near-IR to X-ray data are taken from Fig. 7 and Table 6 of Turner et al. (2002). The IRAS data for Far-IR and Mid-IR flux are derived from the NED database. The luminosity is calculated from observed flux assuming isotropic radiation, zero cosmological constant, deceleration parameter $q_{0}=0.5$, and Hubble constant $H_{0}=75 \mathrm{~km} \mathrm{~s}^{-1} \mathrm{Mpc}^{-1}$. Data are plotted in Fig. 4 as open squares. Ton S 180 has low Galactic and intrinsic extinction (Turner et al. 2002). Since Ton S 180 is a nearby object $(z=0.06)$, we have not taken any spectral shifts or $K$-corrections into account.

The $\mathrm{BH}$ mass estimated from the $\mathrm{H} \beta$ width and optical luminosity (Wandel et al. 1999; Kaspi et al. 2000) is 
Table 1. Best parameter sets for the non self-gravitating disc.

\begin{tabular}{ccccccc}
\hline \hline set & $M_{\mathrm{BH}}\left[M_{\odot}\right]$ & $r_{\mathrm{Sch}}[\mathrm{cm}]$ & $\dot{M}\left[L_{\mathrm{Edd}} / c^{2}\right]$ & $r_{\mathrm{sg}}\left[r_{\mathrm{Sch}}\right]$ & $\Sigma\left[\mathrm{g} / \mathrm{cm}^{2}\right]^{a}$ & $H[\mathrm{~cm}]^{b}$ \\
\hline 1 & $10^{7}$ & $3.0 \times 10^{12}$ & $10^{2.75}$ & 2000 & $2.4 \times 10^{6}$ & $1.2 \times 10^{15}$ \\
2 & $10^{6.8}$ & $1.9 \times 10^{12}$ & 1000 & 3000 & $2.6 \times 10^{6}$ & $1.3 \times 10^{15}$ \\
\hline
\end{tabular}

${ }^{a}$ Half surface density $\Sigma$ at $r=r_{\mathrm{sg}}$.

${ }^{b}$ Semi thickness $H$ at $r=r_{\mathrm{sg}}$.

$10^{7.1} M_{\odot}$. This value is comparable to $10^{7.3} M_{\odot}$ inferred from the [O III] width using Eq. (2) in Wang \& Lu (2001). Its $B$ band luminosity, $v L_{v}(B)$, is about $10^{44.6} \mathrm{erg} / \mathrm{s}$, implying that $\dot{M} \simeq 500 L_{\text {Edd }} / c^{2}$ (see Kawaguchi 2003). Thus, this object is expected to be one of the highest $\dot{M} /\left(L_{\mathrm{Edd}} / c^{2}\right)$ objects among NLS1s and Narrow-Line QSOs.

\subsection{Spectrum of the inner, slim disc}

The structure and emergent spectrum of the inner disc around a non-rotating $\mathrm{BH}$ is computed numerically following the same procedure as in Kawaguchi (2003). The theoretical background of the numerical method is described in Matsumoto et al. (1984) and in Honma et al. (1991a,b). The model employs the standard prescription for turbulent viscosity $v_{\mathrm{t}}=\alpha c_{\mathrm{s}} H$ (Shakura \& Sunyaev 1973) where $c_{\mathrm{s}}$ is the sound speed and $H$ the semi-thickness. The input parameters are $M_{\mathrm{BH}}, \dot{M}$ and viscosity parameter $\alpha$. Given a set of these parameters, we integrate the differential equations for the flow from $2 \times 10^{4} r_{\mathrm{Sch}}$ down to the inner free boundary at $1.01 r_{\mathrm{Sch}}$.

The best parameters for this object have been selected by the least-square procedure, comparing the predicted spectrum (with a hard X-ray power-law due to a corona) to the powerlaw fits for the optical/UV/X-ray spectrum (thick striped lines in Fig. 4; see Table 7 in Turner et al. 2002).

First, the following parameter region is surveyed; $M_{\mathrm{BH}}=$ $10^{6.5-7.5} M_{\odot}, \dot{M}=100-1000 L_{\mathrm{Edd}} / c^{2}$, and $\alpha=0.1-0.001$ with $\Delta \log \left(M_{\mathrm{BH}}\right)=\Delta \log (\dot{M})=0.5$, and $\Delta \log (\alpha)=1$. Among them, one parameter set $\left(M_{\mathrm{BH}}=10^{7} M_{\odot}, \dot{M}=10^{2.5} L_{\mathrm{Edd}} / c^{2}\right.$ and $\alpha=0.001)$ exhibits the best fit. Next, we compute the spectra in more detail around this parameter set, with $\Delta \log \left(M_{\mathrm{BH}}\right)=0.2$, $\Delta \log (\dot{M})=0.25$, and $\Delta \log (\alpha)=0.3$. Finally, we selected two best sets of parameters as listed in Table 1, with $\alpha=0.002$ in both cases. The mass of this inner $\operatorname{disc} M_{\mathrm{disc}}\left(r<r_{\mathrm{sg}}\right)$ is $0.009 \times$ $M_{\mathrm{BH}}$ for set 1, and $0.016 \times M_{\mathrm{BH}}$ for set 2, respectively. Values at $r_{\mathrm{sg}}(\Sigma$ and $H)$ are used as the inner boundary conditions for the self-gravitating part.

The emergent spectrum from $r<r_{\text {sg }}$ (for set 2) is shown in Fig. 4 by a solid line (right), while relevant physical quantities are shown in Fig. 5. For a presentation purpose, a simple $T_{\text {eff }}$ profile (i.e. $T_{\text {eff }} \propto r^{-3 / 4}$ ) is also drawn as a dotted line.

As to the broken power-law description for the X-ray data, different combinations of the photon index $\Gamma$ and breaking energy are deduced for different observations. In order to assess how the different combinations affect the generation of the best sets of parameters, we have also performed the same least-square procedure with the

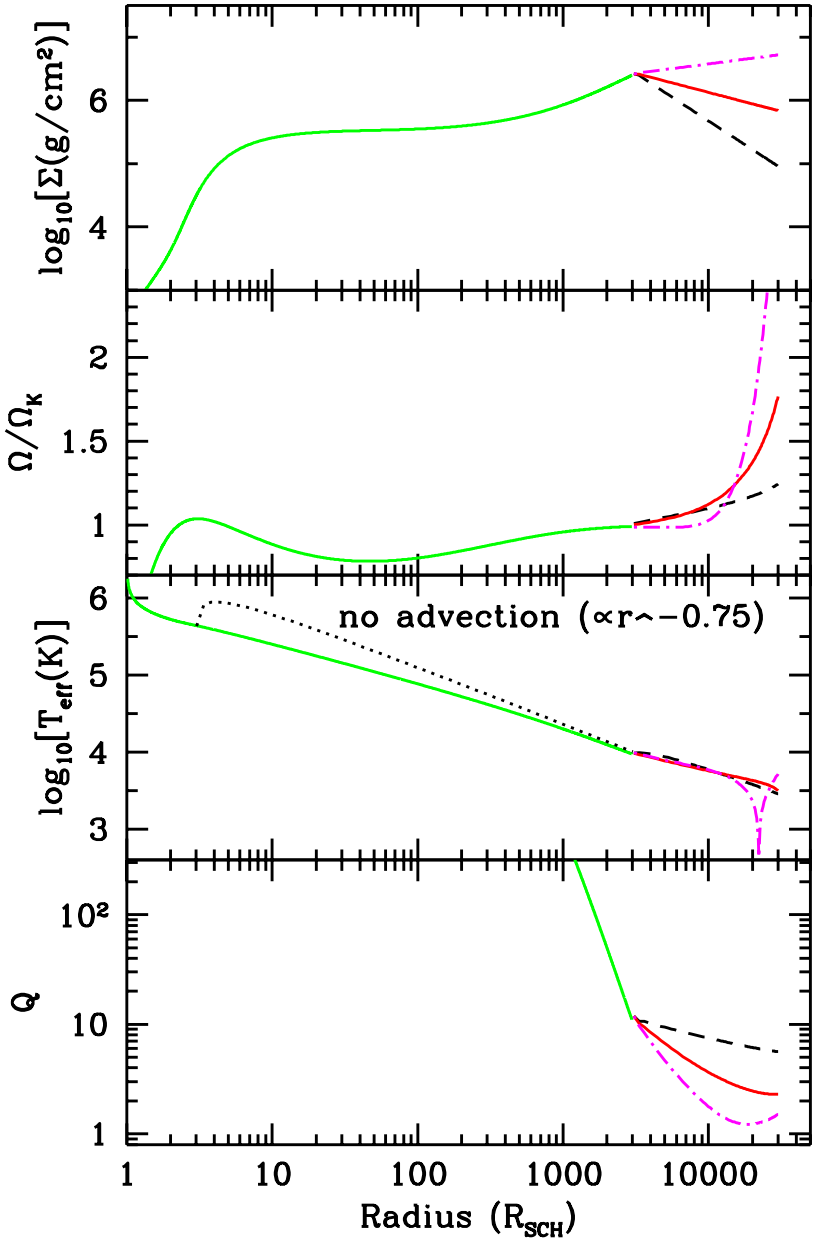

Fig. 5. Variation with radius of some disc parameters (the inner, non self-gravitating part is based on set 2); half surface density $\Sigma$, rotational frequency normalized to the Keplerian value $\Omega / \Omega_{\mathrm{K}}$, effective temperature $T_{\text {eff }}$, and Toomre $Q$-parameter. The solid lines are computed with $\gamma=-0.6$ and $\alpha_{\text {out }}=0.02$, while dot-dashed and dashed ones are computed for $\gamma=0.3$ with a constant $\alpha$ and $\gamma=-1.5$ with $\alpha_{\text {out }}=0.1$, respectively. Two accretion regimes are detached at $r=r_{\mathrm{sg}}\left(=3000 r_{\mathrm{Sch}}\right)$.

X-ray data replaced by the broken power-law description in Vaughan et al. (2002). The following four parameter sets provide best fits; $\left[\log \left(M_{\mathrm{BH}} / M_{\odot}\right), \dot{M} /\left(L_{\mathrm{Edd}} / c^{2}\right), \alpha\right]=$ $(6.8,1000,0.002),(6.8,1000,0.004),(7.0,562,0.002)$, and $(7.2,178,0.002)$. The goodness of the fit to the data shows a slightly shallower distribution than the fit to the Turner et al. (2002) data. In the rest of this study, we emphasize the fit to the data in Turner et al. (2002), where the broadband data were obtained quasi simultaneously.

\subsection{Spectrum of the non-Keplerian, self-gravitating disc}

In the standard theory of radiatively cooled accretion discs (e.g., Pringle 1981), the effective temperature $T_{\text {eff }}$ (close to the surface temperature) is given by

$\sigma T_{\mathrm{eff}}^{4}(r)=v_{\mathrm{t}} \Sigma\left(r \frac{\mathrm{d} \Omega}{\mathrm{d} r}\right)^{2}$ 
where $\Omega$ is the rotation frequency. Again, the turbulent viscosity is chosen to be the standard prescription: $v_{\mathrm{t}}=\alpha c_{\mathrm{s}} H$ (Shakura \& Sunyaev 1973). The sound speed $c_{\mathrm{s}}$ is found from the requirement of hydrostatic equilibrium, namely

$c_{\mathrm{s}}^{2}=\Omega_{\mathrm{K}}^{2} H^{2}+4 \pi G \Sigma H$.

At $r \gtrsim r_{\mathrm{sg}}$, the disc becomes self-gravitating, namely $\Omega$ is expected to depart from its Keplerian value $\Omega_{\mathrm{K}}$. In order to compute $\Omega$, we assume that the rotation curve has only a gravitational origin, that is

$r \Omega^{2}(r)=\frac{G M_{\mathrm{BH}}}{r^{2}}-g_{R}^{\mathrm{disc}}(r)$,

where $g_{R}^{\text {disc }}$ is the contribution due to the whole disc, including the inner slim disc. In general, at large distances, we expect $g_{R}^{\text {disc }}<0$ with the consequence that $\Omega>\Omega_{\mathrm{K}}$. This means a possible reduction of the gradient, $|\mathrm{d} \Omega / \mathrm{d} r|$, and subsequently a change of the effective temperature (Eq. (11)) with respect to a standard disc. It is worth noting that a similar idea has been proposed by Lodato \& Bertin (2001) in order to explain the IR-excess of T Tauri stars.

Since the self-gravitating disc should be gravitationally unstable, other sources of heating, e.g., heating by gravitational instabilities (Adams et al. 1988; Lodato \& Bertin 2001), may overcome the heating by turbulent viscosity (see Lodato \& Rice 2003). To take into account this possibility, we allow the parameter $\alpha$ in the self-gravitating part to increase linearly with $r$, from 0.002 (the value used for the inner disc in Sect. 3.1) at $r_{\mathrm{sg}}$ to $\alpha_{\text {out }}$ at the outermost radius of the self-gravitating disc $r_{\text {out }}$.

Since there is no simple formula for radial self-gravity, we have determined $g_{R}^{\text {disc }}$ numerically using the accurate Poisson 3D-solver, which is described in Huré (2003) for discs with various shapes, sizes and surface density profiles. This has been performed assuming that $H \propto R^{\beta}$ and $\Sigma \propto R^{\gamma}$, where $\beta$ and $\gamma$ are input parameters. Here, $H$ and $\Sigma$ are continuous at the transition between the two regimes of the disc. The radial extension of the self-gravitating disc $r_{\text {out }}$ and the viscosity parameter $\alpha$ at that radius $\alpha_{\text {out }}$ are also input parameters. Those values $(\beta, \gamma$, $r_{\text {out }}$, and $\alpha_{\text {out }}$ ) are determined so that the total spectra (Sect. 3.4) provide acceptable fits to the observed one.

For each model, the effective temperature $T_{\text {eff }}(r)$ is derived from the resultant rotation frequency using Eq. (11). The spectrum of the self-gravitating disc is then computed in the same way as the inner slim disc (i.e., with the effect of electron scattering, even though the effect is small in the outer selfgravitating part).

When we add the spectra of the outer, self-gravitating disc onto the inner disc spectrum, it is found that the combined spectra based on set 2 (Table 1 ) generally provide a better fit to the observed optical/UV spectrum than those based on set 1 . Thus, we hereafter discuss the models obtained for set 2 alone as an example.

For set 2, three possible combinations of parameters are shown in Table 2. The best parameters for $\beta$ and $r_{\text {out }}$ are common to them: $\beta \simeq 1$ and $r_{\text {out }} \simeq 3 \times 10^{4} r_{\text {Sch }}\left(=10 \times r_{\mathrm{sg}}\right)$. For one case $\left(\gamma=-0.6\right.$ and $\left.\alpha_{\text {out }}=0.02\right)$, the spectra from the selfgravitating part (upper dotted line) and the total spectrum (thick dashed line) are presented in Fig. 4. Those three combinations
Table 2. Successful solutions for the self-gravitating disc.

\begin{tabular}{rlc}
\hline \hline$\gamma$ & $\alpha_{\text {out }}$ & $M_{\text {disc }}\left(r \leq r_{\text {out }}\right) / M_{\mathrm{BH}}$ \\
\hline 0.3 & $0.002^{a}$ & 7.0 \\
-0.6 & 0.02 & 1.4 \\
-1.5 & 0.1 & 0.4 \\
\hline
\end{tabular}

${ }^{a}$ Namely, constant $\alpha$ in the entire disc.

exhibit almost identical spectra (deviation of $v L_{v}$ is $0.05 \mathrm{dex}$ at most). Other combinations are also acceptable. The cumulative disc mass $M_{\text {disc }}\left(r \leq r_{\text {out }}\right)$ is not negligible, ranging from $0.4 M_{\mathrm{BH}}$ to $7.0 M_{\mathrm{BH}}$. For the intermediate case, for instance, the total mass, $M_{\mathrm{BH}}+M_{\mathrm{disc}}$, is about $2.4 M_{\mathrm{BH}}=10^{7.2} M_{\odot}$, which is consistent with the central mass estimated by $\mathrm{H} \beta$ and [O III] line widths. It indicates that $M_{\mathrm{BH}}$-estimations using line widths overestimate the $\mathrm{BH}$ mass systematically. To assess how the spectrum changes with different $\gamma$, the total spectrum obtained with $\gamma=0$, which is less preferable, is indicated by the upper dashed line.

Figure 5 presents several physical quantities of the disc for the three successful parameter sets (Table 2) as a function of radius; $\Sigma, \Omega$ (Eq. (13)) and $T_{\text {eff }}$. Vertical optical thickness of the flow, $\tau\left[\tau=\Sigma \times\left(\kappa_{\mathrm{abs}}+\kappa_{\mathrm{es}}\right)>\Sigma \kappa_{\mathrm{es}}=0.4\left(\Sigma / \mathrm{g} \mathrm{cm}^{-2}\right)\right]$ is much larger than unity everywhere. For the case with a very massive $\operatorname{disc}(\gamma=0.3), \Omega$ becomes flat and even starts to increase with radius due to the strong radial self-gravity. This produces a local decrease in $T_{\text {eff }}\left[\propto(\mathrm{d} \Omega / \mathrm{d} r)^{0.5}\right.$; Eq. (11)] at a small range of radius. However, the temperature profiles $T_{\text {eff }}(r)$ for the three cases are mostly the same, and thus they produce almost identical spectra.

\subsection{Emission from the dusty torus}

The dusty torus component is supposed to be responsible for the Mid-IR $(\sim 30 \mu \mathrm{m})$ to $\sim 2 \mu \mathrm{m}$ emission. In general, the Near-IR flux from AGN varies with time following the optical flux with a time lag of about a month-year (e.g., Clavel et al. 1989; Glass 1992; Nelson 1996). This implies that the dusty torus is located quite far from the $\mathrm{BH}$, and that it is heated by the radiation from the vicinity of the central $\mathrm{BH}$.

A detailed modeling of the emission of the torus is beyond the scope of this paper. Here, we adopt a simple power-law description with a cut-off. Theoretical arguments on the dust sublimation as well as observational data (as a spectral bump around $3 \mu \mathrm{m}$ ) indicate that the maximum temperature of the torus is $\sim 1000-1500 \mathrm{~K}$ (e.g. Efstathiou et al. 1995; Kobayashi et al. 1993; Pier \& Krolik 1993). It corresponds to wavelength of $\sim 2.4 \mu \mathrm{m}$ (i.e. $h c /(4 k T)$ with $T=1500 \mathrm{~K}$ ), or $v \simeq 10^{14.1} \mathrm{~Hz}$ (i.e. $4 k T / h)$.

We assume that the fluxes at $25 \mu \mathrm{m}$ and $12 \mu \mathrm{m}$ come from the dusty torus alone, while the near-IR flux is partly due to the self-gravitating disc. Then, the simple model we apply is

$v L_{v}=10^{38.45} v^{0.45} \exp \left(-v / 10^{14.1} \mathrm{~Hz}\right) \mathrm{erg} / \mathrm{s}$. 
We are confident that this is neither the only nor the best possible description. The spectral index inferred for this object differs from the typical mid-IR-near-IR index of bright AGNs $\left(v L_{v} \propto v^{-0.4}\right.$; Neugebauer et al. 1987; Polletta \& Couvoisier 1999; see also Grupe et al. 1998).

We can specify the location $r_{\mathrm{t}}$ of the inner torus (see Fig. 1) by considering that the torus is in thermal balance (heating by radiation from the inner disc is balanced by radiative cooling):

$r_{\mathrm{t}} \simeq 5.3 \times 10^{17}\left(\frac{L}{10^{45} \mathrm{erg} / \mathrm{s}}\right)^{0.5}\left(\frac{T}{1500 \mathrm{~K}}\right)^{-2} \mathrm{~cm}$.

where $L$ and $T$ are the bolometric luminosity of the central disc, and the gas temperature at the innermost radius of the torus, respectively. For the set 2, we have $r_{\mathrm{t}} \simeq 5.3 \times 10^{17} \mathrm{~cm}(=0.17 \mathrm{pc}) \simeq 2.8 \times 10^{5} r_{\mathrm{Sch}}$, which is about 10 times larger than the outer radius of the self-gravitating disc $r_{\text {out }}$. Thus, gravitational attraction on the self-gravitating disc by the torus should not be crucial, unless the torus mass exceeds the $\mathrm{BH}$ mass by some order of magnitude.

\subsection{Mid-IR-X-ray spectral energy distribution}

The thick dashed line in Fig. 4 shows the broad-band, mid-IR to X-ray spectral modeling for Ton S 180, comprised of the three components described in the subsections above. We see that the optical component can be interpreted as the thermal emission from the self-gravitating, non-Keplerian disc. The models for the self-gravitating disc with $\gamma$ and $\alpha_{\text {out }}$ listed in Table 2 provide equally good fits to the observed spectrum. The upper dashed line (for $\gamma=0$ and $\alpha_{\text {out }}=0.02$ ) is drawn to show to what extent a different value of $\gamma$ changes the total spectrum.

If the outer self-gravitating disc is replaced by a Keplerian, standard disc, the resultant total spectrum (lower dashed curve) is not adequate to explain the observed optical/near-IR spectrum. In other words, flux enhancement at the outer region is necessary. This enhancement depends on the resultant rotation law, as well as $\alpha(r)$, at the outer self-gravitating disc.

We demonstrate that a broadband spectrum of one NLS1, Ton S 180, is indeed well fitted by a summation of an inner, non self-gravitating disc, an outer, self-gravitating disc and a dusty torus. Detailed mid-near-IR observations, including temporal studies (e.g. Glass 1992; Nelson 1996), will provide a more accurate model of the dusty torus. Subtraction of the torus component from the total (disc and torus) spectrum will enable us to derive stronger constraints on the size of the selfgravitating part, and mass of the whole disc, etc. High spatial resolution obtained with ground-based, mid- to near-IR interferometers with the large telescopes, such as OHANA (Mariotti et al. 1996; Perrin et al. 2000) and VLTI, will contribute to solving these problems.

\section{Implications and limitations of the present study}

As is obtained in Sect. 3.2, the integrated disc mass is about (0.4-7.0) $M_{\mathrm{BH}}$ (Table 2). Although the Toomre $Q$ parameter $\left[=\Omega c_{\mathrm{s}} /(\pi \Sigma G)\right]$ remains greater than one in the whole disc (Fig. 5), it is quite close to unity at outer radius for larger $\gamma$.
This fact supports the introduction of the additional heating due to gravitational instabilities (e.g., increasing $\alpha$ used here; see Lodato \& Rice 2003). Consensus on the amount of such heating has not yet been reached. Further understanding on this topic, which is related to $\alpha_{\text {out }}$ in this study, will allow us to constrain $\gamma$ (where $\Sigma \propto r^{\gamma}$ ) and the disc mass more severely.

The size of the broad-line region (BLR), $R_{\mathrm{BLR}}$, for Ton $\mathrm{S} 180$ is expected to be of the order of 100 lt.d., using the empirical relation between the optical luminosity and $R_{\mathrm{BLR}}$ (Kaspi et al. 2000). This radius corresponds to $0.085 \mathrm{pc}=2.6 \times$ $10^{17} \mathrm{~cm}$; equivalently, $1.4 \times 10^{5} r_{\mathrm{Sch}}$ for $M_{\mathrm{BH}}=10^{6.8} M_{\odot}$. Thus, the broad-line region is likely located between the inner radius of the torus $\left(r_{\mathrm{t}} \simeq 3 \times 10^{5} r_{\mathrm{Sch}}\right)$ and the outer radius of the selfgravitating disc $\left(r_{\text {out }} \simeq 3 \times 10^{4} r_{\text {Sch }}\right)$. However, the constraint on $r_{\text {out }}$ derived in Sect. 3.2 is rather weak. If the outer massive disc extends at $r \gg r_{\text {out }}$, the clouds in the broad-line region may not be in Keplerian motion, which will lead to an uncertainty for the $M_{\mathrm{BH}}$-estimation with $\mathrm{H} \beta$ width (e.g., Krolik 2001).

Since the main purpose of this paper is to report the discovery of the spectral window for observing a self-gravitating disc, and to demonstrate a spectral fit to the observed spectrum of a super-Eddington accreting AGN, we did not treat the following two issues very accurately: (i) the vertical self-gravity (the last term in the right-hand-side of Eq. (12)) in the inner, non self-gravitating disc, and (ii) the effect of realistic absorption opacity in deriving $\rho_{\text {mid-plane }}$ (Sect. 2.1). Also, possible outflow from the disc is not taken into account either in the inner slim part or in the outer self-gravitating part. Outflow/evaporation at the outer region may contribute to the formation of BLR clouds or broad absorption line (BAL) clouds. These issues will be examined in the future.

\section{Conclusions}

We have presented the outermost radius of the non selfgravitating accretion disc $\left(r_{\mathrm{sg}}\right)$ around a super-massive black hole $(\mathrm{BH})$, i.e. the inner edge of the self-gravitating disc, as a function of the $\mathrm{BH}$ mass $M_{\mathrm{BH}}$, accretion rate $\dot{M}$ and viscosity parameter $\alpha$. We then showed the corresponding wavelength $\left(\lambda_{\mathrm{sg}}\right)$ that is relevant to the emission from $r_{\mathrm{sg}}$.

When $\dot{M} \lesssim 70 L_{\mathrm{Edd}} / c^{2}$ (for $M_{\mathrm{BH}}=10^{7} M_{\odot}$ ), $\lambda_{\mathrm{sg}}$ is roughly proportional to $\dot{M}^{-0.5}$ for fixed $M_{\mathrm{BH}}$ and $\alpha$. With a higher $\dot{M}, \lambda_{\mathrm{sg}}$ reaches and stays at $\sim 4000 \AA$ for $\alpha=0.1$. Interestingly, $\lambda_{\text {sg }}$ is as small as $\sim 1500 \AA$ for the lowest $\alpha$ case in this study (i.e. $\alpha=0.001$ ). Therefore, the continuum emission from $\sim 2 \mu \mathrm{m}$ to $\lambda_{\text {sg }}$ arises from the self-gravitating part of the superEddington accreting disc. Thus, we have discovered a spectral window for observing the self-gravitating disc, which has only been studied by maser spots at the parsec-scale for a few nearby objects.

Next, we demonstrated that the mid-IR to X-ray spectrum of a bright, well-studied Narrow-Line Seyfert 1 galaxy, Ton S 180, is indeed well fitted by the spectrum arising from the following three components: an inner slim disc (with a corona), an outer, self-gravitating non-Keplerian disc and a dusty torus. Comparing the model spectra of the slim disc with the observed UV-X-ray one, the following parameters are favored: $M_{\mathrm{BH}} \approx 10^{6.8} M_{\odot}, \dot{M} \approx 1000 L_{\mathrm{Edd}} / c^{2}$, and $\alpha \approx 0.002$. In the 
model for the outer, self-gravitating disc, we allow the viscosity parameter $\alpha$ to increase with radius, and an outer radius of $3 \times 10^{4} r_{\text {Sch }}$ is inferred. Various profiles for the surface density $\Sigma$ are acceptable, e.g., from $\Sigma \propto r^{0.3}$ to $\Sigma \propto r^{-1.5}$, depending on $\alpha$ at the outermost radius of the disc.

The accretion disc is quite massive, and the disc mass can be (0.4-7.0) $M_{\mathrm{BH}}$. The total mass, BH mass plus the entire disc mass, is found to be about $2.4 M_{\mathrm{BH}}=10^{7.2} M_{\odot}$ if $\Sigma \propto r^{-0.6}$, which is consistent with the central mass estimated by $\mathrm{H} \beta$ and [O III] line widths. This indicates that $M_{\mathrm{BH}}$-estimations using line widths systematically overestimate the $\mathrm{BH}$ mass. Although the disc mass is quite high relative to the $\mathrm{BH}$ mass, the Toomre $Q$ parameter remains greater than one in the whole disc, so that the disc is marginally stable.

Moreover, $r_{\mathrm{sg}}$ can be less than the photon trapping radius for $\dot{M} \gtrsim 10^{3.3} L_{\mathrm{Edd}} / c^{2}$. Thus, a self-gravitating, optically-thick, advection-dominated accretion disc is expected to appear in the extremely high accretion rate regime.

Acknowledgements. We thank Suzy Collin, Helene Sol, Julien Woillez, Catherine Boisson, and Shin Mineshige for useful comments. Detailed and productive comments from the referee, Anuradha Koratkar, are also appreciated. We are deeply grateful to Ryoji Matsumoto and Fumio Honma who developed the numerical code to solve the radial structure of super-Eddington accretion discs. This research has made use of the NASA/IPAC Extragalactic Database (NED) which is operated by the Jet Propulsion Laboratory, California Institute of Technology, under contract with the National Aeronautics and Space Administration. T.K. is supported by the Japan Society for the Promotion of Science (JSPS) Postdoctoral Fellowships for Research Abroad (464).

\section{References}

Abramowicz, M. A., Czerny, B., Lasota, J. P., \& Szuszkiewicz, E. 1988, ApJ, 332, 646

Adams, F. C., Ruden, S. P., \& Shu, F. H. 1989, ApJ, 347, 959

Balluch, M. 1988, A\&A, 200, 58

Begelman, M. C., \& Meier, D. L. 1982, ApJ, 253, 873

Brandt, N., \& Boller, T. 1998, Astron. Nachr., 319, 163

Collin, S., Boisson, C., Mouchet, M., et al. 2002, A\&A, 388, 771

Clavel, J., Wamsteker, W., \& Glass, I. S. 1989, ApJ, 337, 236

Czerny, B., \& Elvis, M. 1987, ApJ, 321, 305

Efstathiou, A., Hough, J. H., \& Young, S. 1995, MNRAS, 277, 1134

Frank, J., King, A., \& Raine, D. 1992, Accretion Power in Astrophysics (Cambridge Univ. Press), second edition

Glass, I. S. 1992, MNRAS, 256, 23

Goldreich, P., \& Lynden-Bell, D. 1965, MNRAS, 130, 97

Grupe, D., Beuermann, K., Thomas, H.-C., Mannheim, K., \& Fink, H. H. 1998, A\&A, 330, 25

Honma, F., Matsumoto, R., \& Kato, S. 1991a, PASJ, 43, 147
Honma, F., Matsumoto, R., Kato, S., \& Abramowicz, M. A. 1991b, PASJ, 43, 261

Hōshi, R., \& Inoue, H. 1988, PASJ, 40, 421

Huré, J.-M. 1998, A\&A, 337, 625

Huré, J.-M. 2002, A\&A, 395, L21

Huré, J.-M. 2003, A\&A, submitted

Kaspi, S., Smith, P. S., Netzer, H., et al. 2000, ApJ, 533, 631

Kato, S., Fukue, J., \& Mineshige, S. 1998, Black-hole accretion disks (Kyoto Univ. Press), Chap. 3

Kawaguchi, T. 2003, ApJ, 593, 69

Kobayashi, Y., Sato, S., Yamashita, T., Shiba, H., \& Takami, H. 1993, ApJ, 404, 94

Koratkar, A., \& Blaes, O. 1999, PASP, 111, 1

Krolik, J. H. 2001, ApJ, 551, 72

Lodato, G., \& Bertin, G. 2001, A\&A, 375, 455

Lodato, G., \& Bertin, G. 2003, A\&A, 398, 517

Lodato, G., \& Rice, W. K. M. 2003, Proc. in Plasmas in the laboratory and in the Universe: new insights and new challenges [astro-ph/0309570]

Madej, J. 1974, Acta Astron., 24, 327

Mariotti, J.-M., Coude Du Foresto, V., Perrin, G., Zhao, P., \& Lena, P. 1996, A\&AS, 116, 381

Matsumoto, R., Kato, S., Fukue, J., \& Okazaki, A. T. 1984, PASJ, 36, 71

Mineshige, S., Kawaguchi, T., Takeuchi, M., \& Hayashida, K. 2000, PASJ, 52, 499

Miyoshi, M., Moran, J., Herrnstein, J., et al. 1995, Nature, 372, 127

Mushotzky, R. F., Done, C., \& Pounds, K. A. 1993, ARA\&A, 31, 717

Nakai, N., Inoue, M., \& Miyoshi, M. 1993, Nature, 361, 45

Nelson, B. O. 1996, ApJ, 465, L87

Neugebauer, G., Green, R. F., Matthews, K., et al. 1987, ApJS, 63, 615

Pier, E. A., \& Krolik, J. H. 1993, ApJ, 418, 673

Perrin, G., Lai, O., Lena, P. J., \& Coude du Foresto, V. 2000, in Interferometry in Optical Astronomy, ed. P. J. Lena, \& A. Quirrenbach, Proc. SPIE, 4006, 708

Polletta, M., \& Courvoisier, T. J.-L. 1999, A\&A, 350, 765

Pringle, J. E. 1981, ARA\&A, 19, 137

Rybicki, G. B., \& Lightman, A. P. 1979, Radiative Processes in Astrophysics (New York: John Willey \& Sons)

Shakura, N. I., \& Sunyaev, R. A. 1973, A\&A, 24, 337

Shimura, T., \& Manmoto, T. 2003, MNRAS, 338, 1013

Shore, S. N., \& White, R. L. 1982, ApJ, 256, 390

Thatte, N., Quirrenbach, A., Genzel, R., Maiolino, R., \& Tecza, M. 1997, ApJ, 490, 238

Telesco, C. M., Becklin, E. E., Wynn-Williams, C. G., \& Harper, D. A. 1984, ApJ, 282, 427

Turner, T. J., Romano, P., Kraemer, S. B., et al. 2002, ApJ, 568, 120

Vaughan, S., Boller, T., Fabian, A. C., et al. 2002, MNRAS, 337, 247

Wandel, A., \& Petrosian, V. 1988, ApJ, 329, L11

Wandel, A., Peterson, B. M., \& Malkan, M. A. 1999, ApJ, 526, 579

Wang, T., \& Lu, Y. 2001, A\&A, 377, 52

Wang, J.-M., \& Netzer, H. 2003, A\&A, 398, 927

Wisotzki, L., Dreizler, S., Engels, D., Fink, H.-H., \& Heber, U. 1995, A\&A, 297, L55 